\title{
Intensity Based Image Registration by Maximization of Mutual Information
}

\author{
R.Suganya, \\ Thiagarajar college of Engg., Madurai, \\ TamilNadu,India \\ Dr.S.Rajaram, \\ Thiagarajar college of Engg.,Madurai, \\ TamilNadu,India
}

\author{
K.Priyadharsini, \\ Thiagarajar college of Engg.,Madurai, \\ TamilNadu,India .
}

\begin{abstract}
:
Biomedical image registration, or geometric alignment of twodimensional and /or three-dimensional (3-D) image data, is becoming increasingly important in diagnosis, treatment planning, functional studies, and computer-guided therapies and in biomedical research [1]. Registration is an important problem and a fundamental task in image processing technique. In the medical image processing fields, some techniques are proposed to find a geometrical transformation that relates the points of an image to their corresponding points of another image. In recent years, multimodality image registration techniques are proposed in the medical imaging field. Especially, CT and MR imaging of the head for diagnosis and surgical planning indicates that physicians and surgeons gain important information from these modalities. In radiotherapy planning manual registration techniques performed on MR image and CT images of the brain. Now-adays, physicians segment the volume of interest (VOIs) from each set of slices manually. However, manual segmentation of the object area may require several hours for analysis. Furthermore, MDCT images and MR images contain more than 100 slices. Therefore, manual segmentation and registration method cannot apply for clinical application in the head CT and MR images. Many automatic and semiautomatic image registration methods have been proposed [2]. The main techniques of image registration are performed by the manual operation, using Landmark and using voxel information. In this paper, an automatic intensity based registration of head images by computer has been employed by applying maximization of mutual information. The primary objective of this paper is to increase accuracy of the registration and reduce the processing time. Experiments show our algorithm is a robust and efficient method which can yield accurate registration results.
\end{abstract}

\section{Keywords:}

Image registration, Mutual information, Medical imaging, Multimodality image.

\section{INTRODUCTION:}

Medical images are becoming increasingly widely used healthcare and in bio-medical research and a very wide range of imaging modalities is now available, such as CT, MRI, PET, SPECT, and so on. In some clinical scenarios, the information from several different imaging modalities should be integrated to deduce useful clinical conclusions. Image registration aligns the images and so establishes correspondence between different features contained on different imaging modalities, allows monitoring of subtle changes in size or intensity over time or across a population and establishes correspondence between images and physical space in image guided interventions.

Image registration is the process of determining the spatial transform that maps points from one image (defined as the moving image) to homologous points on an object in the reference image (called as the fixed image). The similarity of the two images will be calculated and investigated after each transform until they are matched.

Of the multitude of image registration similarity measures that have been proposed over the years, mutual information is currently one of the most intensively researched measures. This attention is a logical consequence of both the favourable characteristics of the measure and the good registration results reported. Mutual information is an automatic, intensity-based metric, which does not require the definition of landmarks or features such as surfaces and can be applied in retrospect [2]. Furthermore, it is one of the few intensity-based measures well suited to registration of multimodal images. Unlike measures 
based on correlation of gray values or differences of gray values, mutual information does not assume a linear relationship among the gray values in the images. However, the mutual information registration function can be well-defined, containing local maxima. Another drawback of MI is that it is calculated on a pixel-by-pixel basis, applying that it tasks into account only the relationship between corresponding individual pixels and not those of each pixel's respective neighbourhood. As a result, much of the global spatial information inherent in images is not utilized. In addition, it is a time-consuming work, especially for high resolution images, because mutual information of the two images must be calculated in each iteration.

In this paper, a novel method for automatic registration of head images by computer, which obtained CT and MR images employing maximization of mutual information, and reduce the processing time.

\section{Medical image datasets}

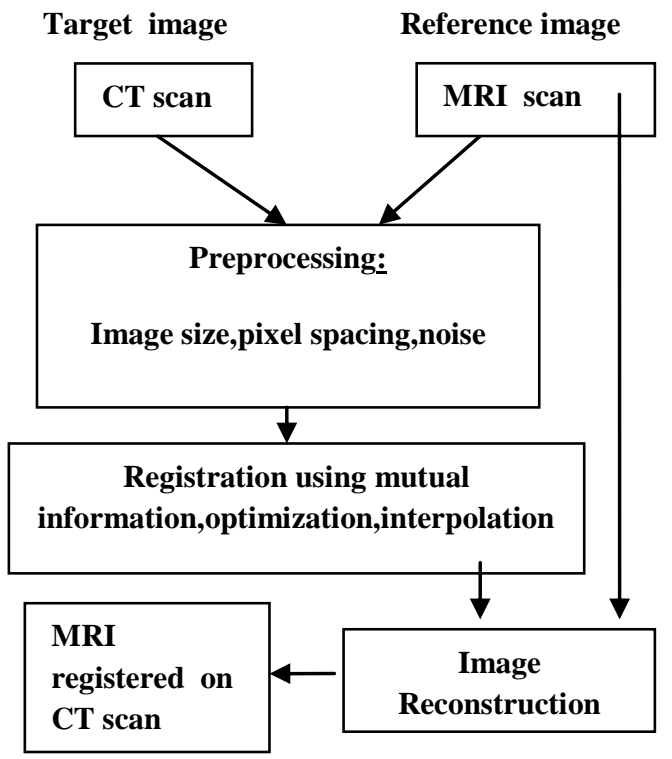

Figure 1: Block diagram for multimodality image registration

\section{MUTUAL INFORMATION AND IMAGE REGISTRATION}

In this paper, we develop a multi-modal image registration technique, which is based on CT and MR imaging of head. When an image superposes onto another one, which obtained multi-modality, several preprocessing techniques are needed for image registration. For multi-modal image registration, the relation between the sizes of image is usually not same. In the preprocessing step, we remove some noise and normalize the size of image. In the next step, initial point is detected by using center of gravity of each image as an initial registration. Finally, the registration is performed employing maximization of mutual information. The details of each method are shown as follows.

\subsection{Pre-processing and initial registration}

The images that obtained from different modalities may have different image size, pixel spacing and a number of slices. In the preprocessing step, image size, pixel spacing is normalized and image noise is reduced for registration. To align the multimodal images, center of gravity is used for initial registration. In this paper, we assume that the MR image is target image and CT image is reference image. In this step, the center of gravity on multi-modal images has been calculated as follows.

Let a digital image be denoted by $f(x, y)$ where $x$ and $y$ are bounded positive integers. The $(i+j){ }^{\text {th }}$ order moment mij of $f(x, y)$ is defined by

$$
\begin{aligned}
& \operatorname{Mij}=\sum x^{j} y^{j} f(x, y) \\
& (x, y) \varepsilon R
\end{aligned}
$$

Where $\mathrm{R}$ is a specified region. The centroid denoted by $\left(\mathrm{x}_{\mathrm{c}}, \mathrm{y}_{\mathrm{c}}\right)$ is calculated by

$\left(\mathrm{x}_{\mathrm{c}}, \mathrm{y}_{\mathrm{c}}\right)=\left(\mathrm{m}_{10} / \mathrm{m}_{00}, \mathrm{~m}_{01} / \mathrm{m}_{00}\right)$

The target image is transformed onto the $\mathrm{x}-\mathrm{y}$ coordinates of the derived center of gravity. It must be the same coordinates as an initial position for registration.

The most popular technique of interpolation is linear interpolation, which defines the intensity of a point as the weighted combination of the intensities of its neighbours.

\subsection{Summation of intensity projection with weight}

In order to reduce the computational time, three dimensional (3-D) image data is projected as a two dimensional (2-D) image data. It is similar to Maximum Intensity Projection (MIP) method which is applied in medical imaging field as 3-D image represent technique. We make the six 2-D image from 3D image set for image registration by calculating the Summation of Intensity Projection (SIP) with weight on each direction. The weight depends on the distance from the screen. $\mathrm{X}_{\mathrm{k}}(\mathrm{k}=1,2, \ldots \mathrm{n})$ is assumed as the change in signal density in 3D space. The expression of SIP with weight describe as follows.

$$
\begin{gathered}
\mathrm{N}=\sum_{\mathrm{K}=1} \mathrm{x}_{\mathrm{k}} \mathrm{w}_{\mathrm{k}} \\
\mathrm{K}=1
\end{gathered}
$$


Where $\mathrm{S}$ is the value of density with projected to screen and wk shows weight value.

\subsection{Final Registration}

Final registration is performed by using the mutual information method. For two images A and B, mutual information I(A,B) can be defined as follows [12].

$\mathrm{I}(\mathrm{A}, \mathrm{B})=\sum \mathrm{p}_{\mathrm{A}, \mathrm{B}}(\mathrm{a}, \mathrm{b}) \log \mathrm{p}_{\mathrm{A}, \mathrm{B}}(\mathrm{a}, \mathrm{b}) / \mathrm{p}_{\mathrm{A}}(\mathrm{a}) \cdot \mathrm{p}_{\mathrm{B}}(\mathrm{b})$

The interpretation of this equation measures the distance between the joint distribution of image pixel value $\mathrm{p}_{\mathrm{A}, \mathrm{B}}(\mathrm{a}, \mathrm{b})$ and the joint distribution in case of independence of the image, $\mathrm{p}_{\mathrm{A}}(\mathrm{a}), \mathrm{p}_{\mathrm{B}}(\mathrm{b})$. In this paper, the target image is transformed for maximization of mutual information. When mutual information is calculated in the images, the intensities of corresponding points of two images $a$ and $b$ are used for making 2-D histogram $h(a, b)$. It can be thought that the probability distribution function can be obtained by dividing the histogram by $\mathrm{N}$, where $\mathrm{N}$ is a number of pixels. It can be expressed as follows:

$$
\begin{gathered}
\mathrm{p}_{\mathrm{A}, \mathrm{B}}(\mathrm{a}, \mathrm{b})=\mathrm{h}(\mathrm{a}, \mathrm{b}) / \mathrm{N} \\
\mathrm{p}_{\mathrm{A}}(\mathrm{a})=\sum \mathrm{p}_{\mathrm{A}, \mathrm{B}}(\mathrm{a}, \mathrm{b}) \\
\mathrm{b} \\
\mathrm{p}_{\mathrm{B}}(\mathrm{b})=\sum \mathrm{p}_{\mathrm{A}, \mathrm{B}}(\mathrm{a}, \mathrm{b})
\end{gathered}
$$$$
\text { a }
$$

When mutual information is detected, the intensity of pixel is linearly converted from 0 to 255 . In this paper, the amount of the movement of the target image is described by optimization that uses multi-dimensional direction set method (powell's method) of which the index is mutual information. This method can be used to 3-D data. But, it needs more computational time. Thus the calculation cost is decreased by using 2-D data of target image and reference image, 2-D data is obtained by the method of SIP with weighted value.

The target image is the CT image and MRI is the reference image. The reference image compare to the target image we get the matched image and rotated image shown in Fig 2, and Fig 3 .

\section{Proposed Algorithm}

PROCEDURE: Image Registration that uses 2-D data

Input: $\mathrm{F}$ and $\mathrm{R}$

Output: Registration using Mutual information
Begin

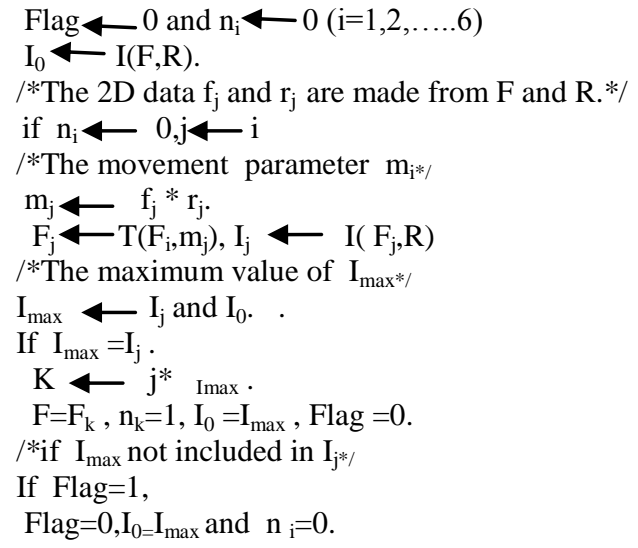

Here I shows the mutual information, F shows target image, R shows reference image, $\mathrm{i}$ shows the number of $2 \mathrm{D}$ data and $\mathrm{T}(\mathrm{F}, \mathrm{m})$ shows the image $\mathrm{F}$ is transformed by using the movement parameter $\mathrm{m}$.

\section{EXPERIMENTAL RESULTS}

The Proposed technique was applied to the two different modalities images- CT and MR images of five human head images and satisfactory results are achieved. The parameters of CT and MR images are shown in table 1 . In this paper, the image that has $512 * 512$ pixels in each slice were converted into $256 * 256$ pixels to reduce the processing time.

The experimental results are shown in Fig 2, Fig 3, table 2.shows the transformation and matching of the image using mutual information.

\section{CONCLUSION}

Multi-modal medical intensity registration is an important capability for finding the correct geometrical transformation that brings one intensity pixel in precise spatial correspondence with another intensity pixel. Multi-modal intensity registration is needed to benefit from the complementing information in medical images of different modalities. The result demonstrates that our registration technique allows fast, accurate, robust and completely automatic registration of multimodality medical images. From the image the value of mutual information in MRI and CT are compared with existing method and shown in Table 2. Compared with the existing method, our experimental results are better with using the intensity of two images.

Table 1.Image parameter

\begin{tabular}{|c|c|c|}
\hline & CT image & MRI image \\
\hline Size[pixels] & $512 * 512$ & $512 * 512$ \\
\hline
\end{tabular}




\begin{tabular}{|c|c|c|}
\hline $\begin{array}{c}\text { Pixel spacing } \\
{[\mathrm{mm}]}\end{array}$ & 0.638 & 0.638 \\
\hline $\begin{array}{c}\text { Slice } \\
\text { thickness[mm] }\end{array}$ & 2 & 2 \\
\hline
\end{tabular}
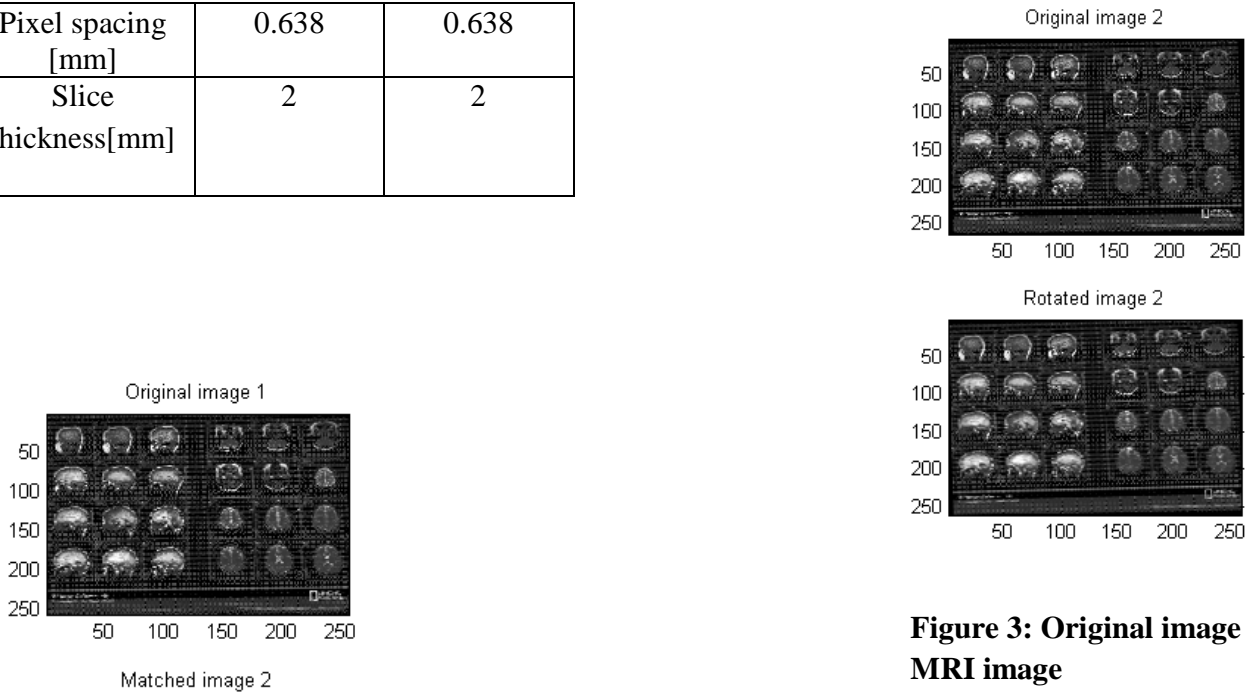

Figure 3: Original image and the corresponding rotated MRI image

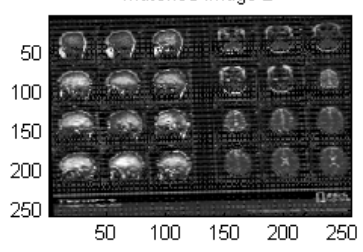

Figure 2: Original image and the corresponding matched MRI image

Table 2: Experimental results

\begin{tabular}{|c|c|c|}
\hline Number & Existing method & Proposed method \\
\cline { 2 - 3 } & Mutual information & Mutual information \\
\hline 1 & 0.4473 & 0.5658 \\
\hline 2 & 0.5174 & 2.5083 \\
\hline 3 & 0.4667 & 2.5396 \\
\hline 4 & 0.5639 & 2.9598 \\
\hline
\end{tabular}

\section{REFERENCES}

[1] L. Ding, A. Goshtasby, M. Satter, "Volume image registration by template matching", Image and Vision Computing, Vol.19, No.12, pp.821-832,2001.

[2] J. M. Fitzpatrick, D. L. G. Hill, Y. Shyr et al.,"Visual Assessment of the Accuracy of
Retrospective Registration of MR and CT Images of the Brain", IEEE Trans. on Medical Image., Vol.17, No.4, pp.571$585,1998$.

[3] Friston KJ, Ashbuner J, Frith CD et al., "Spatial registration and normalization of images", HumanBrain Mapping, Vol. 3, pp.165-189, 1995.

[4] T. Gaens, F. Maes, D. Vandermeulen, P. Suetens,"Nonrigid Multimodal Image Registration Using Mutual 
Information", Intl. Conf on Medical Image Computing and Computer-Assisted Intervention,

Pp.1099-1106, 2003..

[5] B. Kim, J. Boes, K. Frey et al., "Mutual information for automated unwarping of rat brain autoradiographs", Neuro Image, Vol. 5, No. 1, pp. 31-40, 2004.

[6] S. Klinski, C. Derz, D. Weese et al., "Model based image processing using snakes and mutual information", Medical Imaging: Image Processing,Proc. SPIE, pp. 1053-1064, 2000.

[7] D.Mattes, D.R.Haynor, H.Vesselle, T.K. Lewellen, and W.Eubank, "Non-rigid multimodality image registration," Medical Imaging 2001: Image Processing, pp 1609-1620, 2001.

[8] F. Maes, A. Collignon, D. Vandermeulen et al,"Multimodality Image Registration by

Maximization of Mutual Information", IEEE Trans.on Medical Image., Vol.16, No.2, pp.187-198, 1997.

[9] J. Maintz, E. Meijering, M. Viergever, "General multimodal elastic registration based on mutual information", Medical Imaging: Image Processing, Proc. SPIE, pp. 144-154, 1998.
[10] J.P.W.Pluim, J.B.A. Maintz, and M.A.Viergever, "Image Registration by Maximization of Combined Mutual Information and Gradient Information," IEEE Transactions on Medical Imaging

[11] J.P.W.Pluim, J.B.A. Maintz, and M.A.Viergever, "Mutual Information-based registration of medical images:a survey, "Medical Imaging. IEEE Transactions on, vol.22,pp.986-1004, 2003.

[12] A. Roche, G.Malandain, X. Pennec et al., "The Correlation Ratio as a New Similarity Measure for Multimodal Image Registration", Intl. Conf on Medical Image Computing and Computer-Assisted Intervention, pp.1115-1124, 1998.

[13] Wu.A,Hartov.A,Paulsen.D,Roberts.W， "Multimodal Image Registration via Mutual information to account for intilal tissue motion during image-guided neurosurgery". Intl. Conf of the IEEE EMBS Pp.1675-1678, 2005.

[14] B.Zitovaa and J.Flusser, "Image Registration Methods: a survey," Image and Vision Computing, vol.21, pp.977-1000, 2003. 